\title{
Molecular and biological profile may discriminate between synchronous or metachronous endometrial and ovarian cancer
}

1:

Valentina lacobelli

Dipartimento Scienze della vita e sanità pubblica, Università Cattolica del Sacro Cuore, Rome, Italy, Rome, Italy

Pathologist:

Gian Franco Zannoni

Dipartimento Scienze della vita e sanità pubblica, Università Cattolica del Sacro Cuore, Rome, Italy, Rome, Italy

Dipartimento della salute della donna, del bambino e di sanità pubblica, Fondazione Policlinico Universitario A. Gemelli IRCCS, Rome, Italy, Rome, Italy

Radiologist:

Benedetta Gui

Dipartimento Diagnostica per immagini, radioterapia, oncologia e ematologia, Fondazione Policlinico Universitario A. Gemelli IRCCS, Rome, Italy

Discussants:

- Additional material is published online only. To view please visit the journal online (http://dx.doi.org/10.1136/ijgc2020-001545).

\section{Correspondence to}

Professor Francesco Fanfani, Dipartimento della salute della donna, del bambino e di sanità pubblica., Fondazione Policlinico Universitario A. Gemelli IRCCS, Rome, Italy, Rome, Ml 20123, Italy; francesco.fanfani74@ gmail.com

Accepted 30 April 2020 Published Online First 9 June 2020

Check for updates

(c) IGCS and ESGO 2020. No commercial re-use. See rights and permissions. Published by BMJ.

\begin{tabular}{|l|}
\hline To cite: lacobelli V, \\
Zannoni GF, Gui B, et al. \\
Int J Gynecol Cancer \\
2020;30:1071-1076. \\
\hline
\end{tabular}

Anna Fagotti

Dipartimento Scienze della vita e sanità pubblica, Università Cattolica del Sacro Cuore, Rome, Italy, Rome, Italy

Dipartimento della salute della donna, del bambino e di sanità pubblica, Fondazione Policlinico Universitario A. Gemelli IRCCS, Rome, Italy, Rome, Italy

\section{Discussants:}

Giovanni Scambia

Dipartimento Scienze della vita e sanità pubblica, Università Cattolica del Sacro Cuore, Rome, Italy, Rome, Italy

Dipartimento della salute della donna, del bambino e di sanità pubblica, Fondazione Policlinico Universitario A. Gemelli IRCCS, Rome, Italy, Rome, Italy

Discussants:

Francesco Fanfani Dipartimento Scienze della vita e sanità pubblica, Università Cattolica del Sacro Cuore, Rome, Italy, Rome, Italy

Dipartimento della salute della donna, del bambino e di sanità pubblica, Fondazione Policlinico Universitario A. Gemelli IRCCS, Rome, Italy, Rome, Italy

\section{CASE PRESENTATION}

A 42-year-old woman with abnormal vaginal bleeding was referred to the Department of Obstetrics and Gynecology of the Fondazione Policlinico Universitario A. Gemelli, IRCCS in Rome, in June 2019, with a diagnosis of complex hyperplasia with atypia versus well-differentiated endometrioid adenocarcinoma of the uterus. Family history was significant for paternal colorectal cancer, maternal breast cancer, and one grandmother with pancreatic cancer. Her past medical and surgical history was unremarkable, apart from a cesarean section for breech presentation in 2011.

On presentation, her pelvic examination showed a moderately enlarged anteverted mobile uterus, without palpable masses in the adnexal regions. A pelvic ultrasound was obtained. Serum tumor markers 

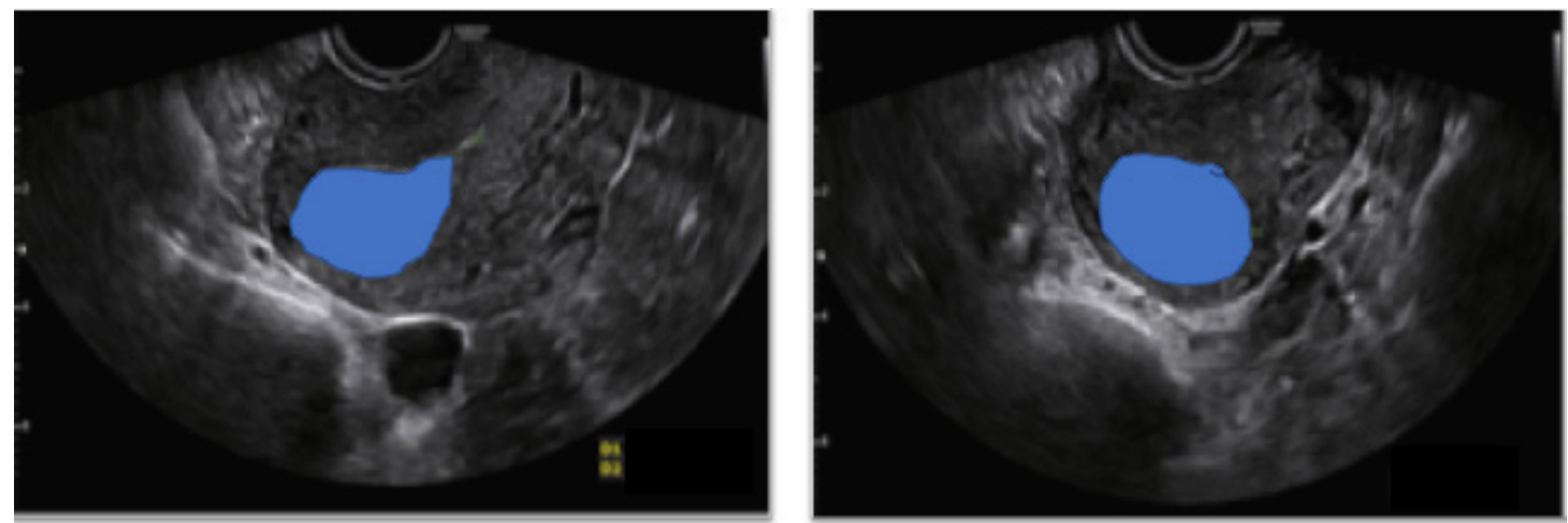

Figure 1 (A-B) Ultrasound revealed a thickened endometrium with the presence of hyperechogenic newly formed tissue, infiltrating $>50 \%$ of the myometrium border, without cervical infiltration.

were: CA125 $18 \mathrm{U} / \mathrm{mL}$ (negative $<35 \mathrm{U} / \mathrm{mL}$ ), HE4 $55 \mathrm{pmol} / \mathrm{L}$ (negative $<70 \mathrm{pmol} / \mathrm{L}$ ), CA19.9 $11 \mathrm{U} / \mathrm{mL}$ (negative $<40 \mathrm{U} / \mathrm{mL}$ ), and CEA $1.8 \mathrm{ng} / \mathrm{mL}$ (negative $<3.0 \mathrm{ng} / \mathrm{mL}$ ).

\section{DR GUI}

On transvaginal ultrasound, the uterus measured $9.5 \times 4.9 \times 4.0$ $\mathrm{cm}$ in length, width, and height, respectively. The examination revealed thickened endometrium with a solid formation of $2.9 \times 2.7$ $\mathrm{cm}$, with myometrial invasion $>50 \%$ and no cervical involvement (Figure 1A-B). The ovaries were normal. Magnetic resonance imaging (MRI) showed a moderate hyperintense lesion within the endometrium in the sagittal and axial T2-weighted images. The lesion obliterated the underline junctional zone in the left anterolateral side indicating myometrial invasion for less than $50 \%$ of its thickness (Figure 2A-B). The cervix and ovaries were normal.
The patient was consented for surgery via a minimally invasive approach. At laparoscopy, there was no evidence of extrauterine disease. A total hysterectomy, bilateral salpingo-oophorectomy, bilateral sentinel lymph node dissection, and peritoneal washing were performed. Operative time and estimated blood loss were $75 \mathrm{~min}$ and $<50 \mathrm{~mL}$, respectively. Surgery was completed without complications. The patient was discharged from hospital 2 days after the operation and no post-operative complications were noted.

\section{DR ZANNONI}

The final pathological findings of the uterus showed an endometrial endometrioid adenocarcinoma grade 2 (Figure 3A), tumor size 2.5 $\mathrm{cm}$, myometrial invasion $3 \mathrm{~mm}$ of $16 \mathrm{~mm}$ without lymphovascular space invasion, negative surgical margins, and no cervical invasion. There was also evidence of a right ovarian mixed endometrioid

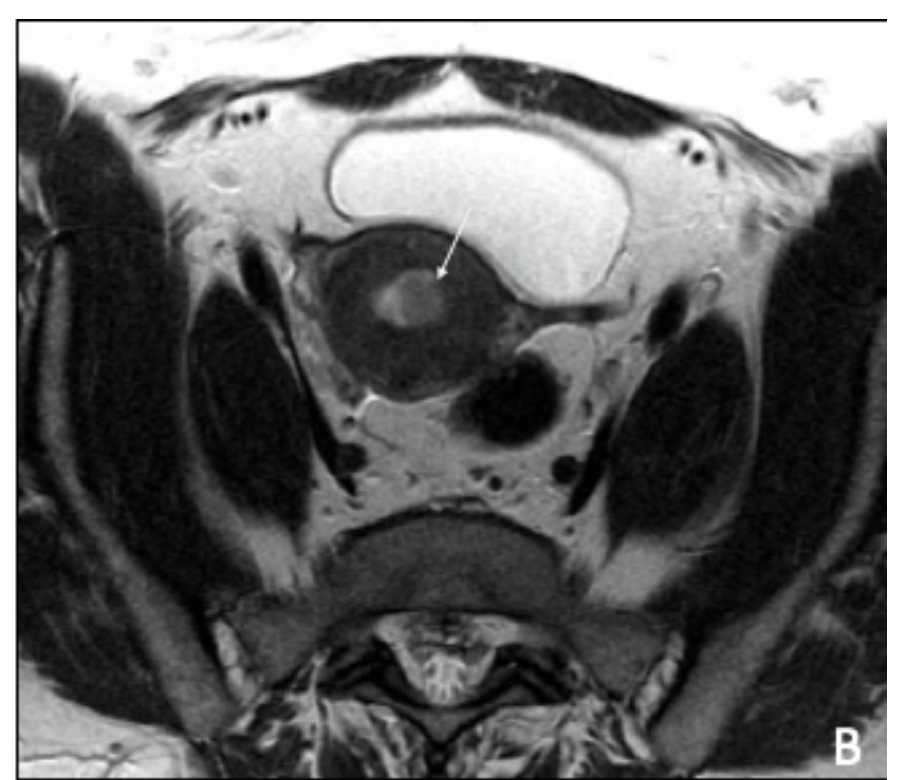

(B) The lesion obliterated the underline junctional zone in the left antero-lateral side (arrow) indicating myometrial invasion for $<50 \%$ of its thickness. 

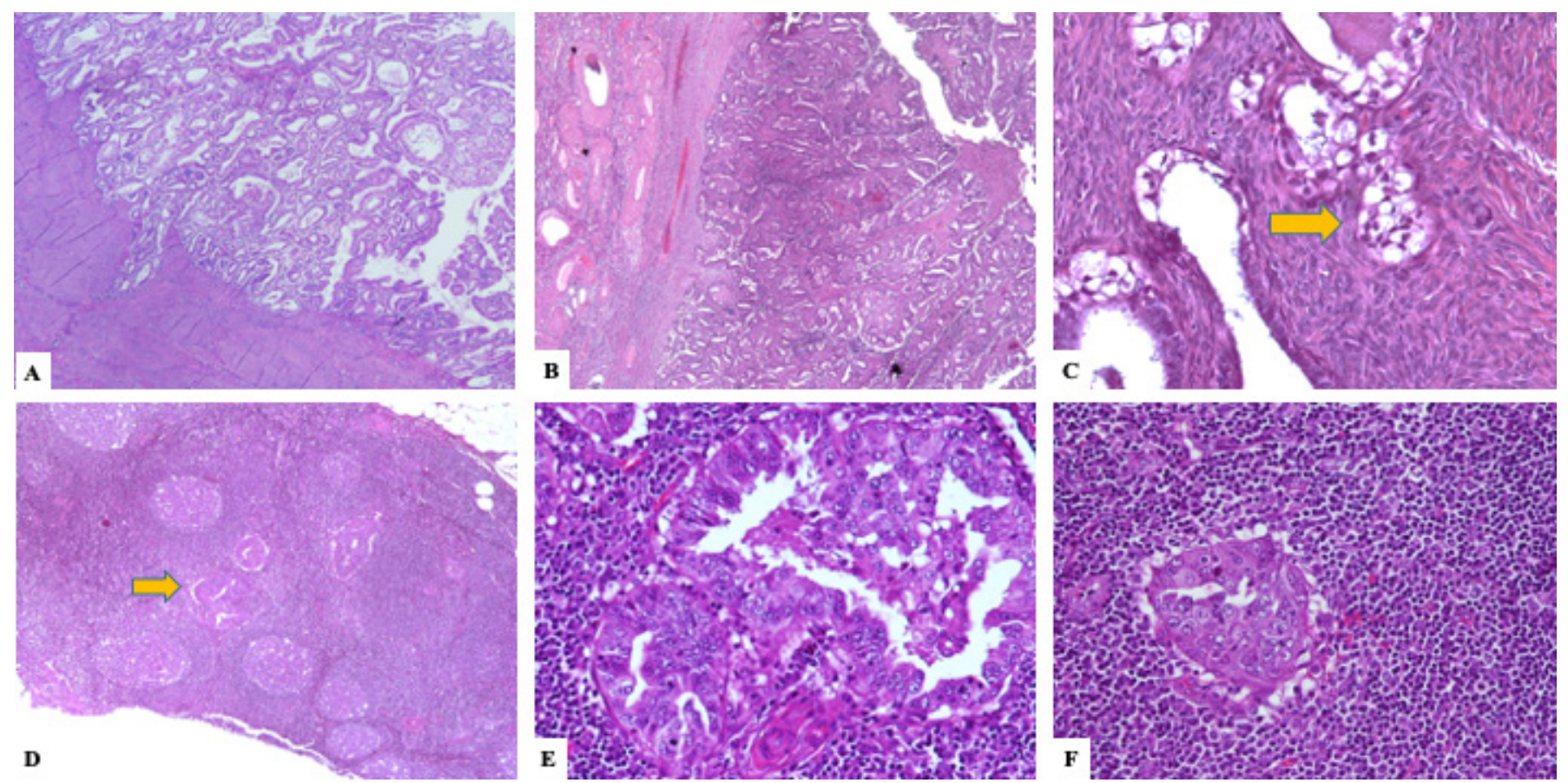

Figure 3 (A) Endometrial neoplasm composed of well-formed endometrial glandular structures consistent with endometrioid endometrial carcinoma G2. (B) Ovarian neoplasm with similar histological features observed in the endometrium. (C) The ovarian neoplasm showed focal areas in which neoplastic cells exhibited clear cell morphology (arrow). These areas were interpreted as a minority clear cell carcinoma component within the ovarian endometrioid adenocarcinoma G2. (D) Metastatic spread within the pelvic lymph is depicted (arrow). (E) Higher-power photomicrograph showing metastatic neoplastic glands with endometrioid morphology. (F) A micrometastasis (arrow) within another pelvic lymph node is depicted.

adenocarcinoma grade 2 (Figure 3B) with a minor component of clear cell carcinoma and a left ovarian clear cell carcinoma of 0.5 $\mathrm{cm}$ (Figure 3C). One right and one left external iliac sentinel nodes were negative on ultrastaging analysis (Online supplementary table 1). All three neoplasms were positive for MLH1 and MLH2, Ioss of expression of MSH2 and MSH6 (unstable immunophenotype), $E R=1+80 \%, P R=2+40 \%$, and $p 53$ wild-type.

The two possible diagnoses were International Federation of Gynecology and Obstetrics (FIGO) stage IIIA endometrial endometrioid adenocarcinoma with ovarian metastasis or synchronous FIGO stage IA ovarian endometrioid and clear cell carcinoma plus stage IA endometrial endometrioid adenocarcinoma.

\section{DR ZANNONI}

Would you please provide details regarding the role of immunohistochemistry and microsatellite instability testing for screening patients with endometrial cancer at risk for hereditary nonpolyposis colorectal cancer (HNPCC) (Lynch syndrome)?

MSH2 and MSH6 gene mutations are mainly responsible for endometrial cancer in Lynch syndrome, while MSH2 is mainly related to ovarian cancer. ${ }^{1}$ Even if available data are limited, it is likely that the same mutations in the MSH2 and MSH6 genes could be the principal cause also of serous epithelial ovarian cancer, as these are the most common genetic alterations found in these two neoplasms. ${ }^{2}$

To date, the most complete molecular study of endometrial cancer has been The Cancer Genome Atlas (TCGA) project, which included a combination of whole-genome sequencing, exome sequencing, microsatellite instability assays, and copy-number analysis. Molecular information was analyzed to divide 232 endometrioid and serous endometrial cancers into four classes - polymerase epsilon (POLE) ultra-mutated, microsatellite instability hypermutated, copy-number low, and copy-number high - that correlate with progression-free survival. ${ }^{3}$

In the setting of our patient, microsatellite instability originates from mistakes in the post-replicative DNA mismatch repair system. Those deficiencies can result from the following: (1) a familiar inherited cancer syndrome (Lynch syndrome), (2) acquired/somatic mutations, or (3) epigenetic events, such as methylation of one of the genes involved in mismatch DNA repair, most commonly MLH1.

All mismatch repair deficiencies (MSH6, PMS2, MLH1, and MSH2) are generally considered together, the age of the specific lifetime risk of Lynch-associated cancers can vary largely according to which gene is mutated: it has been observed that MSH6 mutations are associated with markedly lower cancer risks than MLH1 or MSH2 mutations. ${ }^{4}$ Moreover, according to the specific mismatch repair gene mutation or protein loss identified, the prognostic and predictive implications may differ. Nevertheless, the greatest experience on this subject is derived from over 1000 women with endometrial cancer with tumors evaluated for microsatellite instability, MLH1 methylation, and mismatch repair protein expression by the NRG Oncology/Gynecologic Oncology Group Study (GOG210). Subgroups of normal mismatch repair, epigenetic defect, and probable mutation (somatic or germline) were correlated to clinicopathological variables and clinical outcomes. Their results showed no direct correlation between a subgroup and progression-free and overall survival, highlighting that the molecular profile of endometrial cancer is not yet completely understood. ${ }^{5}$ 


\section{Case study}

In the present case, the low stage of the uterine neoplasia, the absence of lymphovascular space invasion, the association of right ovarian cancer with endometriosis, and the presence of a clear cell component, though minimal, favored the probability of synchronous tumors. Conversely, the bilateral ovarian involvement and the simultaneous microsatellite instability in the three tumors supported the suggestion of a metastatic uterine tumor in which an ovarian component is composed of clear cells, which instead are not present in the endometrial lesion.

Therefore, after a multidisciplinary team discussion, the recommendation was to proceed with positron emission tomography/ computed tomography (PET/CT) scan imaging and consideration of surgical staging for an early ovarian cancer. The PET/CT scan showed no evidence of disease.

\section{DR FANFANI}

\section{Please provide details as to why the patient was} recommended for a second surgery at this time?

The choice to perform full lymph node staging was taken considering two hypotheses: (1) in the case of primary clear cell ovarian cancer, the risk of occult retroperitoneal metastasis is almost $10 \%,{ }^{6}$ and such tumors have a low likelihood of responding to adjuvant chemotherapy and (2) according to NCCN guidelines (version 1.2020) a sentinel lymph node approach can be considered for the surgical staging of apparent uterine-confined disease when there is no metastasis demonstrated by imaging studies or no obvious extrauterine disease at exploration. In this case, the fact that the ovaries were positive for disease was considered an indication for full nodal dissection according to the increased risk of nodal metastases in patients with stage IIIA endometrial cancer compared with early-stage disease.

In September 2019, the patient underwent laparoscopic pelvic and para-aortic lymphadenectomy, omentectomy, and peritoneal washing. The operative time was $95 \mathrm{~min}$ and the estimated blood loss was $150 \mathrm{~mL}$. Final pathology findings showed the presence of one micrometastasis $(0.06 \mathrm{~cm})$ and one macrometastasis $(0.4 \mathrm{~cm})$ in 2/19 right pelvic lymph nodes (Figure 3D-F). A total of 14 left pelvic lymph nodes and 29 para-aortic lymph nodes were negative for disease. No ultrastaging was performed. Omentum and peritoneal washing were also negative for disease (0nline supplementary table 1). The patient was discharged from hospital 2 days after the operation and no post-operative complications were noted.

The case was discussed again at the multidisciplinary team meeting, and on review of final pathology of the lymph nodes it was concluded that nodal metastases were consistent with endometrioid histology. Therefore, the patient was diagnosed with FIGO stage IIIC1 endometrial cancer, and was recommended to undergo six cycles of adjuvant chemotherapy with paclitaxel $\left(175 \mathrm{mg} / \mathrm{m}^{2}\right)$ and carboplatin (AUC 6) followed by external beam radiotherapy. She completed the treatment on December 30, 2019. As of February 2020, the patient is being followed without evidence of disease.

\section{DRS SCAMBIA AND FAGOTTI}

\section{What would you discuss with the patient regarding the best} available adjuvant treatment?

High-risk disease in endometrial cancer is defined as endometrioid endometrial cancer stage I, grade 3 with deep invasion, stage II or III endometrioid endometrial cancer with no residual disease, or non-endometrioid (serous or clear cell) histology, and occurs in approximately 15\%-20\% of cases. For decades, standard adjuvant treatment for women with high-risk endometrial cancer has been pelvic external beam radiotherapy; however, more recently greater emphasis has been given to the use of adjuvant chemotherapy with or without pelvic radiation, as this might improve survival by reducing the risk of metastatic disease. Nevertheless, a number of randomized trials comparing adjuvant chemotherapy with external beam radiotherapy have failed to show an improvement in progression-free survival or overall survival. ${ }^{78}$ However, the recent updated analysis of PORTEC-3, an open-label, multicentre, randomized intergroup phase 3 trial, showed that the combination of adjuvant chemotherapy and radiotherapy for high-risk endometrial cancer significantly improved overall survival and failure-free survival versus radiotherapy alone, especially for women with stage III endometrial cancer. ${ }^{9}$ This study enrolled 660 patients with high-risk endometrial cancer who were randomly assigned to two arms to receive either pelvic radiotherapy alone or the combination of radiotherapy with two cycles of concurrent cisplatin followed by four cycles of adjuvant paclitaxel/carboplatin. At a median follow-up of 72.6 months, 5-year overall survival was $81.4 \%$ with chemoradiotherapy versus $76.1 \%$ with radiotherapy alone (adjusted HR 0.70, $p=0.034$ ). The 5-year failure-free survival was $76.5 \%$ versus $69.1 \%$, respectively (HR $0.70, p=0.016$ ). The first sites of recurrence were distant metastases in most patients with recurrent disease, occurring in 78/330 patients resulting in a 5 -year probability of $21.4 \%$ in the chemoradiotherapy group versus $98 / 330$ patients (5-year probability of $29.1 \%$ ) in the radiotherapy-alone group ( $H R 0.74, p=0.047$ ). At 5 years, grade 3 adverse events were similar for the two groups, occurring in $8 \%$ of 201 patients in the chemoradiotherapy group versus $5 \%$ of 187 patients in the radiotherapy-alone group, while grade 2 or worse adverse events were reported in a significantly higher number of patients in the chemoradiotherapy group $(p=0.002)$. The patient presented here had a mixed endometrioid and clear cell histology, and the analysis of this group in the PORTEC trial found that the frequency of recurrence among women with clear cell cancers was similar to that of women with endometrioid tumors and lower than that of women with serous cancers. Therefore, a combined chemoradiotherapy adjuvant treatment seemed the best option for the patient.

\section{Dr Fanfani}

What is the frequency of Lynch syndrome among women with synchronous primary cancers of the endometrium and ovary? The co-occurrence of adenocarcinoma in the uterus and ovary is found in approximately $5 \%$ of endometrial cancer patients and $10 \%$ of ovarian cancer patients. Most of these are of endometrioid histology. The differentiation between the presence of two separate tumors, ovarian and endometrial, and metastatic disease has an important prognostic and therapeutic significance. ${ }^{10}$ However, despite the adoption of several clinical and biological criteria to stratify low- and high-risk patients, the differential diagnosis between metastatic and independent primary tumors remains diagnostically challenging. ${ }^{311}$ Overall, 
synchronous endometrial and ovarian cancer is a rare finding, but it generally affects women at a young age, presenting at an early stage, being low grade in nature, and with a favorable prognosis compared with endometrial or ovarian cancers alone.

Lynch syndrome occurs in $0.4 \%-1.0 \%$ of ovarian cancers and in $2 \%-6 \%$ of endometrial cancers. ${ }^{5}$ In women with Lynch syndrome, the combined lifetime risks of developing ovarian and endometrial cancer are $10 \%-15 \%$ and $28 \%-60 \%$, respectively. ${ }^{12}$ Overall, the risk of synchronous endometrial and ovarian cancer in the frame of this familial condition is uncertain, although it has been suggested that $\sim 3 \%-14 \%$ of synchronous endometrial and ovarian cancer cases exhibit an association with Lynch syndrome. ${ }^{2}$ This patient presented a family history consistent with Lynch-associated cancers, leading to the suspicion that her disease was a synchronous or metachronous Lynch-associated cancer. She had an endometrioid carcinoma component in the endometrial and ovarian cancers, in line with a previous report suggesting that synchronous endometrial and ovarian cancer tend to include endometrioid histology in both cancers. Moreover, the majority of clinically diagnosed cases with synchronous endometrial and ovarian cancer harbor similar molecular alterations; therefore, they are clonally-related cancers, which probably reflects dissemination from one site to the other.

In this regard, Schultheis et al ${ }^{13}$ reported a series of 17 sporadic synchronous endometrioid endometrial carcinomas and endometrioid ovarian carcinomas and one Lynch syndrome case analyzed by parallel sequencing. In the Lynch syndrome case, the endometrioid endometrial carcinomas and the endometrioid ovarian carcinomas had different somatic mutations, while sporadic samples revealed remarkable correlation between the two neoplasms. Their data suggested that in patients with sporadic synchronous endometrioid endometrial carcinomas and endometrioid ovarian carcinomas the tumors are closely related and likely represent dissemination from one site to the other. However, the patient presented here had an incidental finding of right ovarian mixed endometrioid grade 2 with a minor component of clear cell carcinoma and left ovarian clear cell carcinoma, which led to the recommendation to restage for ovarian cancer. Interestingly, the restaging surgery demonstrated positive pelvic lymph nodes, therefore questioning the former diagnosis. In fact, the positive right pelvic lymph node had endometrioid histology, contradicting the hypothesis of a primitive ovarian cancer and establishing the endometrial origin. This finding is consistent with the recent paradigm-shifting concept that most synchronous endometrial and ovarian cancers originate from a single tumor. ${ }^{13}$

\section{CLOSING SUMMARY}

The case presented here of a young woman diagnosed with endometrial cancer with mismatch repair deficiency shows how challenging the decision algorithm can be when surgical, pathological, and molecular aspects are considered together. In fact, the first not-completely-defining pathological findings (two primary synchronous tumors of ovaries and endometrium vs metastatic endometrial cancer) associated with simultaneous microsatellite instability in all three neoplasms and the endometrioid histology of one of the ovarian lesions, guided us towards further surgical steps. Unexpectedly, the pathology reports from the subsequent surgery revealed two right pelvic lymph node metastases of endometrioid histology, reversing the first hypothesis of an early-stage tumor. This final histological finding led us to the recommendation of adjuvant treatment, as we staged the tumor as endometrial cancer FIGO stage IIIC1.

In conclusion, Lynch syndrome in synchronous endometrial and ovarian cancer is not common, but is more often detected in synchronous endometrial and ovarian cancer compared with general endometrial cancer cases. Given that universal screening for endometrial cancer is becoming standard practice, and that patients with synchronous endometrial and ovarian cancer would be at high risk of Lynch syndrome, it is necessary to perform microsatellite instability or immunohistochemistry analysis in all cases of synchronous endometrial and ovarian cancer in order to better tailor the most appropriate treatment for ovarian or endometrial cancer. The association between microsatellite instability and clinical outcome in endometrial cancer is still controversial, and the prognostic significance of microsatellite instability is yet to be assessed, since only a few studies have reported these data. We believe that epigenetic and genetic mismatch repair analyzes will soon be available, providing innovative information for precision medicine in endometrial carcinomas.

Contributors VI: presenter. GFZ: pathologist. BG: radiologist. AF, GS, FF: discussants.

Funding The authors have not declared a specific grant for this research from any funding agency in the public, commercial or not-for-profit sectors.

Competing interests None declared.

Patient consent for publication Obtained.

Provenance and peer review Commissioned; externally peer reviewed.

\section{REFERENCES}

1 Helder-Woolderink JM, Blok EA, Vasen HFA, et al. Ovarian cancer in Lynch syndrome; a systematic review. Eur J Cancer 2016:55:65-73.

2 Soliman PT, Broaddus RR, Schmeler KM, et al. Women with synchronous primary cancers of the endometrium and ovary: do they have Lynch syndrome? J Clin Oncol 2005;23:9344-50.

3 Talhouk A, McAlpine JN. New classification of endometrial cancers: the development and potential applications of genomic-based classification in research and clinical care. Gynecol Oncol Res Pract 2016;3.

4 Bonadona V, Bonaïti B, Olschwang S, et al. Cancer risks associated with germline mutations in $\mathrm{MLH} 1, \mathrm{MSH} 2$, and MSH6 genes in Lynch syndrome. JAMA 2011;305:2304-10.

5 Goodfellow PJ, Billingsley CC, Lankes HA, et al. Combined microsatellite instability, MLH1 methylation analysis, and immunohistochemistry for Lynch syndrome screening in endometrial cancers from GOG210: an NRG Oncology and Gynecologic Oncology Group study. J Clin Oncol 2015;33:4301-8.

6 Powless CA, Aletti GD, Bakkum-Gamez JN, et al. Risk factors for lymph node metastasis in apparent early-stage epithelial ovarian cancer: implications for surgical staging. Gynecol Oncol 2011;122:536-40.

7 Matei D, Filiaci VL, Randall M, et al. A randomized phase III trial of cisplatin and tumor volume directed irradiation followed by carboplatin and paclitaxel vs. carboplatin and paclitaxel for optimally debulked, advanced endometrial carcinoma. J Clin Oncol 2017;35:5505.

8 Susumu N, Sagae S, Udagawa Y, et al. Randomized phase III trial of pelvic radiotherapy versus cisplatin-based combined chemotherapy in patients with intermediate- and high-risk endometrial cancer: a Japanese Gynecologic Oncology Group study. Gynecol Oncol 2008;108:226-33. 


\section{Case study}

9 de Boer SM, Powell ME, Mileshkin L, et al. Adjuvant chemoradiotherapy versus radiotherapy alone in women with highrisk endometrial cancer (PORTEC-3): patterns of recurrence and post-hoc survival analysis of a randomised phase 3 trial. Lancet Oncol 2019;20:1273-85.

10 Soliman PT, Slomovitz BM, Broaddus RR, et al. Synchronous primary cancers of the endometrium and ovary: a single institution review of 84 cases. Gynecol Oncol 2004;94:456-62.
11 Vermij L, Smit V, Nout R, et al. Incorporation of molecular characteristics into endometrial cancer management. Histopathology 2020;76:52-63.

12 Watson P, Lynch HT. Cancer risk in mismatch repair gene mutation carriers. Fam Cancer 2001;1:57-60.

13 Schultheis AM, Ng CKY, De Filippo MR, et al. Massively parallel sequencing-based clonality analysis of synchronous endometrioid endometrial and ovarian carcinomas. J Natl Cancer Inst 2016;108:djv427. 